\title{
Proceso de molturación mecánica en medio seco, húmedo y criogénico de polvo de hierro dúctil nanoestructurado $\left.{ }^{\circ}\right)$
}

\author{
N. Cinca*, E. Hurtado*, I. G. Cano* y J.M. Guilemany*
}

\begin{abstract}
Resumen
El principal objetivo de este estudio, es obtener una efectiva reducción del tamaño de partícula y de grano de un polvo de hierro nanoestructurado mediante la molturación mecánica en diferentes medios de molienda. La principal dificultad radica en la gran ductilidad del material. Las variables a estudiar en el proceso han sido la velocidad de rotación, la relación polvo/bolas y el porcentaje de agente de control añadido. El polvo se ha estudiado por difracción laser para caracterizar la distribución de tamaños de partículas, microscopía electrónica de barrido (SEM), microscopía electrónica de transmisión (TEM) y difracción de Rayos X. Del estudio comparativo se destaca: la molienda en seco ha dado lugar a una reducción más efectiva tanto del tamaño de partícula (43\%) como del tamaño de grano (62\%), mientras que en condiciones húmedas se ha conseguido disminuir la cantidad de óxido, así como obtener una distribución más homogénea del polvo resultante, aunque la reducción del tamaño de partícula no ha sido tan efectiva; finalmente, los resultados en medio criogénico producen reducciones en tamaños en tiempos más cortos y por tanto resultan prometedores.
\end{abstract}

Palabras clave Molturación mecánica; Deformación plástica; Nanoestructura; DRX; Microscopía electrónica.

\section{Mechanical milling of a nanostructured ductile iron powder under dry, wet and cryogenic atmospheres}

\begin{abstract}
The main objective of this study, is to obtain an effective particle and grain size reduction of a nanostructured iron powder by mechanical milling under different milling media. One of the main challenges in this study is to work with this material of great ductility.The variables of the study to be optimized have been the following: speed of rotation, powder to ball ratio (PBR) and the percentage of control agent to induce an effective powder fracturing in front of cold welding. The powder has been characterized by a Laser Diffraction Particle Size Analyser, scanning electron microscopy (SEM) and transmission electron microscopy (TEM) and, X-ray diffraction.Through the comparative study, it is found that operating under dry milling conditions: there is a more effective particle size reduction of $43 \%$ and grain size reduction of $62 \%$. In wet conditions has been reduced the amount of oxide, as well as to obtain a more homogenous distribution of the resulting powder. The results under cryogenic media is presented as promising.
\end{abstract}

Keywords

Mechanical milling; Plastic deformation; Nanostructure; XRD; Scanning electron microscopy.

\section{INTRODUCCIÓN}

En las últimas décadas ha habido un gran auge en el interés por el estudio de materiales nanoestructurados, es decir, aquellos con tamaños de grano inferior a los 100 nanómetros ( $\mathrm{nm}$ ). Dichas investigaciones han aportado importantes mejoras a nivel de dureza y resistencia entre otras propiedades. Existen distintos métodos para conseguir disminuir el tamaño de grano, entre ellos la molienda mecánica, donde se fractura el material, consiguiendo finalmente partículas con tamaños de grano inferiores a los iniciales pudiendo llegar al nivel nanocristalino.

En la molturación mecánica, los sólidos son reducidos de tamaño por la aplicación de fuerzas de impacto, compresión, cizalla (abrasión) y/o cortado pudiendo aumentar su reactividad y produciendo además una mejora a nivel de dureza y resistencia al reducir el tamaño de grano.

Es posible postular algunas hipótesis para explicar porqué es difícil moler materiales a tamaños pequeños de forma rápida y económica ${ }^{[1]}$. En primer

\footnotetext{
(•) Trabajo recibido el día 18 de Mayo de 2010 y aceptado en su forma final el día 13 de Octubre de 2010.

* Centre de Projecció Tèrmica (CPT). Universitat de BarcelonaMartí i Franqués 1, E-08028, Barcelona, Spain.

e-mail: ncinca@cptub.org; ehurtado@cptub.org; igcano@cptub.org; jmguilemany@cptub.org.
} 
lugar, con el progreso de molienda, la rotura ocurre a partir de las grietas contenidas en las partículas; los fragmentos más pequeños producidos, tienen claramente una menor probabilidad de contener defectos y se aproximan a una resistencia ideal. En segundo lugar, es obvio que cada vez es más difícil capturar las partículas para tensionarlas a medida que se van reduciendo. En tercer lugar, la presencia de partículas muy pequeñas en la masa de polvo puede afectar la capacidad de los medios de molienda para producir un buen impacto a cualquiera de las partículas contenidas en el molino. En cuarto lugar, es posible que las fuerzas cohesivas que existen entre partículas muy pequeñas, le impartan propiedades especiales al lecho como un todo, de tal manera que todas las partículas estarán bien situadas para recibir un impacto. Finalmente, es posible que pequeñas partículas en contacto bajo un gran esfuerzo, puedan volver a reintegrarse.

En cuanto al campo de las nanopartículas, para el caso del hierro, material con el que se realizará la experimentación, ha habido poco interés en sus efectos ópti$\cos ^{[15]}$, sin embargo tanto las propiedades químicas como magnéticas han sido ampliamente estudiadas en varios trabajos $^{[2-5]}$.

En el caso de la vertiente de materiales nanoestructurados, materiales base hierro tanto amorfos como nanocristalinos, han sido investigados para aplicaciones tales como en telecomunicaciones, electrónica, microdispositivos, etc. ${ }^{[6 \text { y } 7]}$, así como por la mejora de sus propiedades mecánicas ${ }^{[8}$ y 9$]$. En general, cuando éstos se sintetizan mediante procesos de molienda mecánica, la gran limitación es evitar que el tamaño de grano aumente durante su posterior consolidación, en cuyo caso se perderían las propiedades que le confiere el reducido tamaño de grano ${ }^{[10]}$.

La mayoría de trabajos de molturación están realizados con equipos de alta energía.
El objetivo del presente trabajo, es comparar la molturación mecánica en tres medios distintos (seco, húmedo y criogénico) de polvo de hierro nanoestructurado y estudiar su influencia en el tamaño de grano y de partícula alcanzados.

\section{DESARROLLO EXPERIMENTAL}

\subsection{Material de partida}

En la figura 1 se puede observar la morfología del polvo de hierro inicial. La tabla I muestra su composición y sus características principales; éste tiene un tamaño medio de partícula de 5,6 $\pm 2,5 \mu \mathrm{m}\left(\mathrm{d}_{90}=11,6\right)$.

El agente de control usado ha sido ácido esteárico en la molturación en seco y etanol para la molturación en húmedo.

Tabla I. Características del polvo de partida

Table I. Feedstock powder

\begin{tabular}{ll}
\hline Densidad aparente & $\sim 3,8 \mathrm{t} / \mathrm{m}^{3}$ \\
\hline Tamaño medio de partícula & $4-6 \mu \mathrm{m}$ \\
Hierro & $>99,5 \%$ \\
Carbono & $<0,05 \%$ \\
Nitrógeno & $<0,01 \%$ \\
Oxígeno & $<0,2 \%$ \\
Níquel & $<100 \mathrm{ppm}$ \\
Cromo & $<150 \mathrm{ppm}$ \\
Molibdeno & $<20 \mathrm{ppm}$ \\
\hline
\end{tabular}

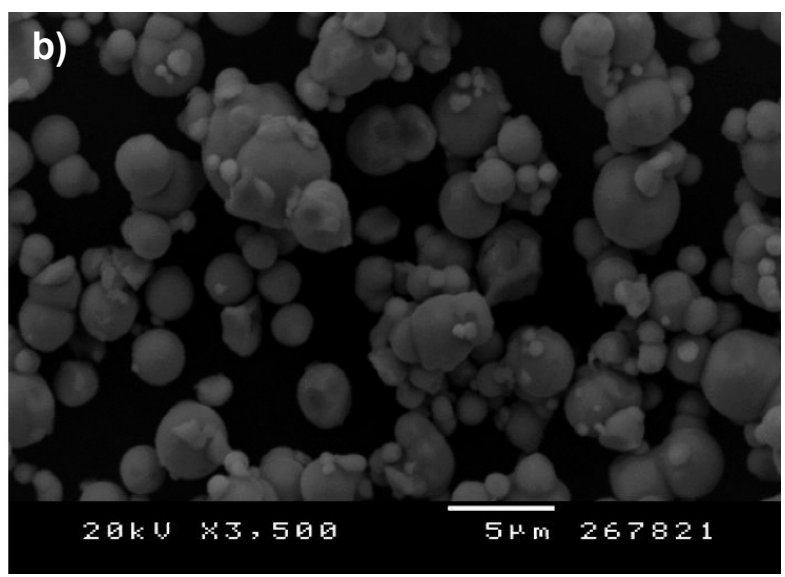

Figura 1. Imágenes de SEM de la morfología del polvo de hierro inicial.

Figure 1. SEM micrographs of the feedstock powder. 
PROCESO DE MOLTURACIÓN MECÁNICA EN MEDIO SECO, HÚMEDO Y CRIOGÉNICO DE POLVO DE HIERRO DÚCTIL NANOESTRUCTURADO MECHANICAL MILLING OF A NANOSTRUCTURED DUCTILE IRON POWDER UNDER DRY, WET AND CRYOGENIC ATMOSPHERES

Para la caracterización del polvo se ha utilizado difracción láser para el análisis de tamaño de partícula (Coulter LS 13320), difracción de Rayos X SIEMENS D-500 Diffractometer, microscopía electrónico de Transmisión TEM JEOL JEM 2100 y, microscopía electrónico de Barrido SEM JEOL JSM-5310.

\subsection{Procedimiento experimental}

Se ha utilizado un molino attritor de la marca UNION PROCESS modelo 01HD, capaz de operar en medio seco, húmedo y criogénico. Los materiales tanto del recipiente de molienda, como bolas y eje son de acero inoxidable AISI 316.

Para la molturación seca se ha realizado un diseño factorial de experimentos $2^{3}$; las variables a estudiar y el planteamiento de experimentos, se indican en las tablas II y III respectivamente.

Para la molturación húmeda se utiliza etanol, añadiéndolo hasta llegar a cubrir las bolas. Por tanto, en este caso se tienen dos variables a estudiar, porque la tercera queda fijada. Escogiendo los mismos niveles que para la molienda seca, el planteamiento del trabajo experimental se muestra también en la tabla III.

Para la molturación criogénica, y tras varios experimentos iniciales, se demostró que las mejores condiciones de operación son: $600 \mathrm{rpm}, \mathrm{PBR}=1: 20$ y 0,5\% de ácido esteárico. Por tanto, conocidos estos factores y debido a los elevados costes de operación se trabajó únicamente en estas condiciones.

\section{RESULTADOS Y DISCUSIÓN}

\subsection{Molienda seca}

Tras la realización de los experimentos definidos en la tabla III, se obtuvo el menor tamaño de partícula, tras 12 h de molturación seca en el caso del

Tabla II. Definición de los factores y sus niveles a estudiar

Table II. Variables design and levels of study

DOMINIO EXPERIMENTAL

\begin{tabular}{cccc}
\hline & FACTORES & Nivel (-) & Nivel (+) \\
\hline $\mathrm{X}_{1}$ & Velocidad (rpm) & 450 & 600 \\
$\mathrm{X}_{2}$ & PBR & $1: 35$ & $1: 20$ \\
$\mathrm{X}_{3}$ & Ácido esteárico (\%) & 0 & 0,5 \\
\hline
\end{tabular}

Tabla III. Diseño experimental a realizar en molienda seca

Table III. Design of experiment: mechanical milling for a dry atmosphere

\begin{tabular}{ccccl}
\hline & $\begin{array}{c}N^{0} \text { de } \\
\text { experimento }\end{array}$ & $\begin{array}{c}\text { Velocidad } \\
(\mathrm{rpm})\end{array}$ & PBR & $\begin{array}{c}\text { Ác. Esteárico } \\
(\%)\end{array}$ \\
\hline Molienda & 1 & 450 & $1: 35$ & 0 \\
en seco & 2 & 600 & $1: 35$ & 0 \\
& 3 & 450 & $1: 20$ & 0 \\
& 4 & 600 & $1: 20$ & 0 \\
& 5 & 450 & $1: 35$ & 0,5 \\
& 6 & 600 & $1: 35$ & 0,5 \\
& 7 & 450 & $1: 20$ & 0,5 \\
Molienda & 8 & 600 & $1: 20$ & 0,5 \\
en húmedo & 1 & 450 & $1: 35$ & - \\
& 2 & 600 & $1: 35$ & - \\
& 3 & 450 & $1: 20$ & - \\
& 4 & 600 & $1: 20$ & - \\
\hline
\end{tabular}

experimento 6: alta velocidad, baja PBR y alto contenido de agente de control, tal y como se extrae del resumen de resultados mostrados en la tabla IV. Se alcanzó un tamaño medio de partícula de 2,0 $\pm 2,3 \mu \mathrm{m}$, consiguiendo, por tanto una reducción del $43 \%$ respecto al tamaño inicial.

En la tabla $\mathrm{V}$ se muestra, entre otros valores estadísticos, el $\mathrm{d}_{90}$ y el $\mathrm{d}_{10}$ (tamaño por debajo del cual se encuentran el 90 y el 10\% de las partículas) del experimento 6; el valor $\mathrm{d}_{90}$ se ha usado como más representativo de la muestra que el valor medio. Analizando

Tabla IV. Tamaños de partícula y de grano de los experimentos de molienda seca

Table IV. Particle size and grain size of the dry milling experiments

\begin{tabular}{lcc}
\hline & $\begin{array}{c}\text { Tamaño de } \\
\text { partícula, } \mathbf{d}_{90} \\
(\mu \mathrm{m})\end{array}$ & $\begin{array}{c}\text { Tamaño de } \\
\text { grano } \\
\text { calculado }(\mathrm{nm})\end{array}$ \\
\hline Muestra inicial & 11,6 & 64,98 \\
Experimento 1 & 33,5 & 62,11 \\
Experimento 2 & 16,3 & 43,29 \\
Experimento 3 & 48,9 & 27,74 \\
Experimento 4 & 40,4 & 37,18 \\
Experimento 5 & 14,2 & 24,94 \\
Experimento 6 & 6,6 & 24,63 \\
Experimento 7 & 43,4 & 39,02 \\
Experimento 8 & 18,9 & 84,63 \\
\hline
\end{tabular}


Tabla V. Datos estadísticos del tamaño de partícula del polvo molturado en seco, tras las condiciones establecidas por el experimento 6 (comparación con el inicial)

Table V. Particle size characteristics of the mechanically milled powder in dry conditions set in the experiment 6 (comparison with the initial state)

\begin{tabular}{lcc}
\hline & $\begin{array}{c}\text { Inicial } \\
(\mu \mathrm{m})\end{array}$ & $\begin{array}{c}\text { Final }(\mathbf{1 2} \mathbf{h}) \\
(\mu \mathrm{m})\end{array}$ \\
\hline Media & 5,063 & 2,012 \\
Desviación estándar & 2,463 & 2,334 \\
$\mathbf{d}_{\mathbf{1 0}}$ & 2,444 & 0,680 \\
$\mathbf{d}_{\mathbf{9 0}}$ & 11,58 & 6,579 \\
\hline
\end{tabular}

muestras a distintos tiempos, se observó una primera fase de soldadura fría provocada por la elevada ductilidad del material, en la que va aumentando el tamaño de partícula conforme va avanzando el tiempo de molturación. Al alcanzar 6 h de molturación y por la acción del ácido esteárico como agente de control, empieza a estar presente la fractura, produciendo una fuerte disminución del tamaño de partícula, hasta llegar a conseguir tras 12 h, un tamaño final inferior al inicial.

En la figura 2 a) se observan partículas mucho más finas que las iniciales, mostradas en la figura 1 . A bajos aumentos se ven agregados de partículas, en las que únicamente a mayores aumentos se pueden diferenciar las partículas entre sí. En este caso la soldadura es mínima y se ve muy favorecida la fractura ${ }^{[11]}$

En la figura $2 \mathrm{~b}$ ) se puede intuir el perfil de algunos de los granos, e incluso la orientación de los planos cristalinos en muchos de ellos. En todos los granos observados, los tamaños observados se encuentran entre 8 y $19 \mathrm{~nm}$. Se ha encontrado un tamaño de grano promedio de 11,8 $\pm 3,9 \mathrm{~nm}$.

Los difractogramas de la muestra de polvo de hierro inicial y la obtenida tras el experimento 6, (Fig. 3), indicaron que se ha producido un importante ensanchamiento de los picos, así como una disminución de las intensidades de todos ellos. Además, aparece un pico adicional identificado como $\mathrm{Fe}_{3} \mathrm{O}_{4}(9 \%$ en peso).

Mediante el método de Williamson-Hall se han calculado los tamaños de grano estimados (Tabla IV). Para la muestra inicial de hierro resulta un tamaño de grano de $64,98 \mathrm{~nm}$, mientras que para la obtenida tras el experimento 6 , resulta ser de $24,63 \mathrm{~nm}$, por lo que las condiciones de dicho experimento dan lugar a una disminución tanto de tamaño de partícula como de grano.

\subsection{Molienda húmeda}

Según los experimentos definidos en la tabla III, se obtuvo el menor tamaño de partícula tras $12 \mathrm{~h}$ de molturación húmeda para los parámetros definidos según el experimento 4: alta velocidad y alta PBR (Tabla VI). No obstante, no se consiguió una efectiva reducción del tamaño de partícula. Sin embargo, las distribuciones de tamaños de partícula han sido
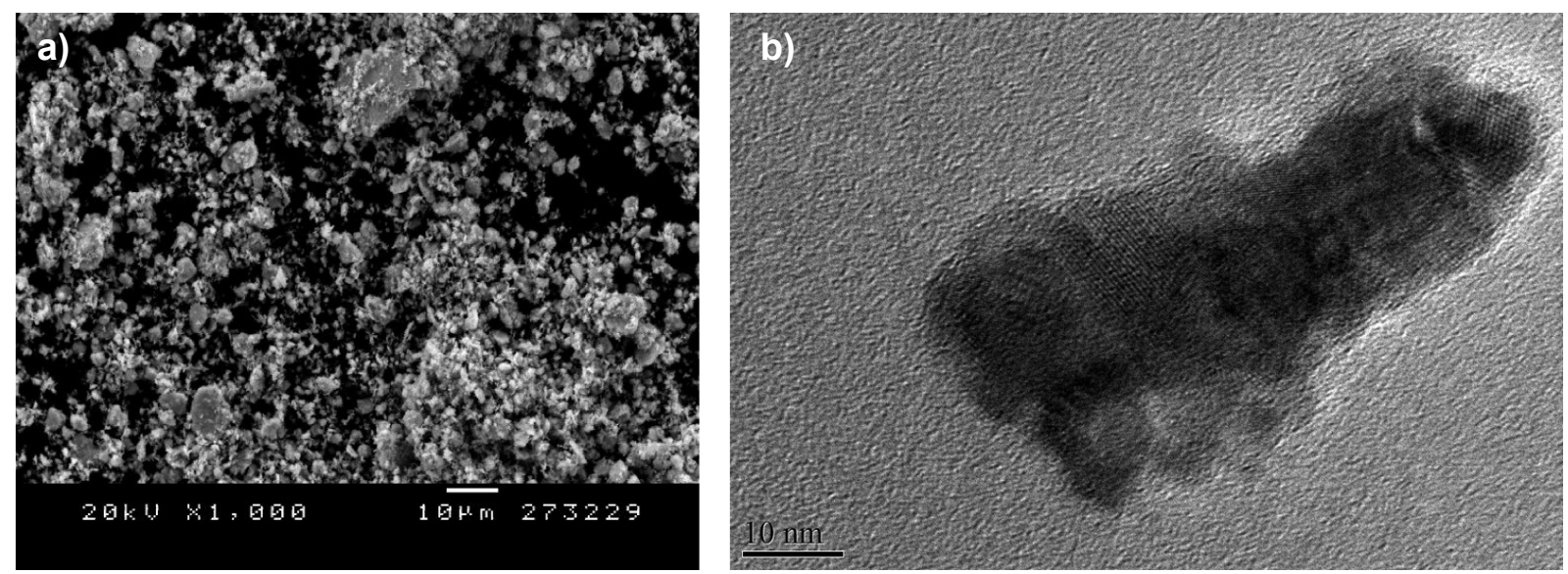

Figura 2. Morfología del polvo molturado en seco tras $12 \mathrm{~h}$, según condiciones del experimento 6; a) SEM y b) TEM.

Figure 2. Powder morphology of the dry mechanically milled powder after $12 h$ according to the $6^{\text {th }}$ experiment set: a) SEM and b) TEM. 
PROCESO DE MOLTURACIÓN MECÁNICA EN MEDIO SECO, HÚMEDO Y CRIOGÉNICO DE POLVO DE HIERRO DÚCTIL NANOESTRUCTURADO MECHANICAL MILLING OF A NANOSTRUCTURED DUCTILE IRON POWDER UNDER DRY, WET AND CRYOGENIC ATMOSPHERES

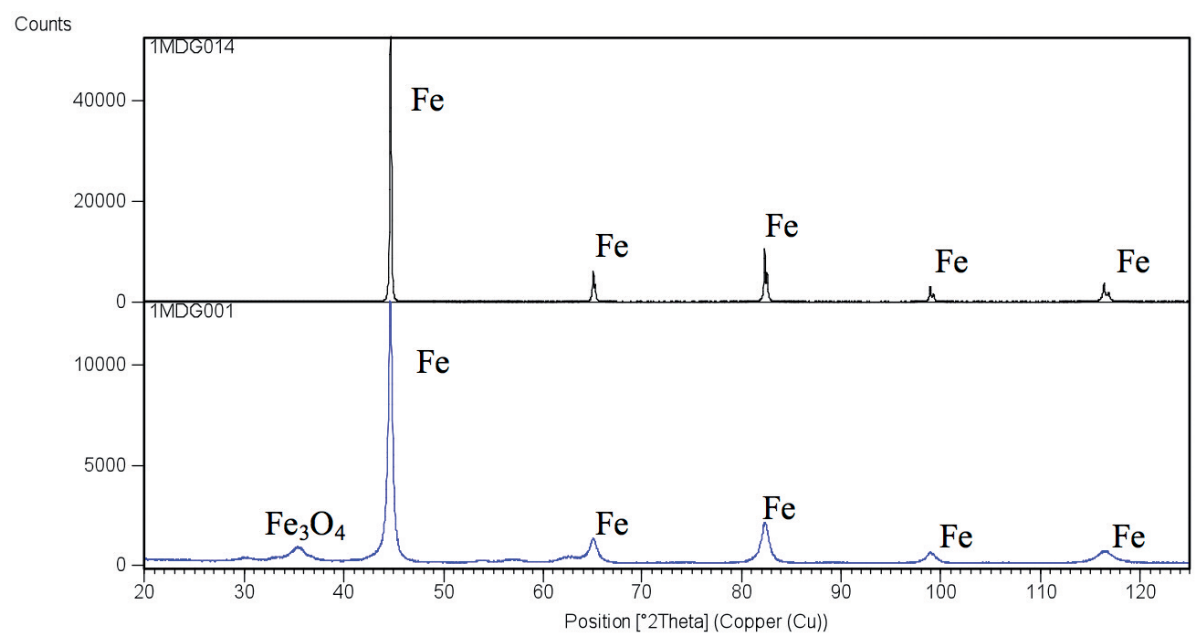

Figura 3. Comparación de los difractogramas obtenidos de la muestra inicial (superior) y la mejor muestra obtenida de la molturación seca (inferior).

Figure 3. X-Ray pattern comparison between the unmilled (up) and dry milled (down) Fe powder.

más estrechas, por tanto, la molienda húmeda homogeneíza la muestra.

De acuerdo con la tabla VII, se observa que el tamaño de partícula final obtenido es muy superior al inicial. De hecho, no es de extrañar, que la molienda húmeda no sea tan efectiva como la seca en cuanto a reducción de tamaño de partícula, si se considera que una suspensión de polvo fino en agua es altamente viscosa. La naturaleza viscosa de la suspensión, absorbe (amortigua) la fuerza de impacto, produciendo una menor fractura de las partículas en la suspensión.

En la figura 4 a), se observan partículas excesivamente deformadas y muy aplanadas. En este caso, ha

Tabla VI. Tamaños de partícula y de grano de los experimentos de molienda húmeda

Table VI. Particle size and grain size of the wet milling experiments

\begin{tabular}{lcc}
\hline & $\begin{array}{c}\text { Tamaño de } \\
\text { partícula, } d_{90} \\
(\mu \mathrm{m})\end{array}$ & $\begin{array}{c}\text { Tamaño de } \\
\text { grano } \\
\text { calculado }(\mathrm{nm})\end{array}$ \\
\hline Muestra inicial & 11,6 & 64,98 \\
Experimento 1 & 85,0 & 31,25 \\
Experimento 2 & 74,9 & 49,50 \\
Experimento 3 & 75,2 & 53,19 \\
Experimento 4 & 69,9 & 52,59
\end{tabular}

habido mucha contribución de soldadura fría y muy poca de fractura. Por microscopía de transmisión, se observan algunos límites de granos bien diferenciados, además de las orientaciones de los planos cristalinos. En todos los casos, los granos observados en todas las imágenes obtenidas por TEM, se encuentran entre 9 y $29 \mathrm{~nm}$ y realizando un promedio de todas las medidas realizadas, el tamaño de grano promedio obtenido tras la molturación húmeda es de 16,5 $\pm 5,9 \mathrm{~nm}$.

El difractograma de la muestra obtenida tras el experimento 4, también mostró un ensanchamiento

Tabla VII. Datos estadísticos del tamaño de partícula del polvo molturado en húmedo, tras las condiciones establecidas por el experimento 4 (comparación con el inicial)

Table VII. Particle size characteristics of the mechanically milled powder, in wet conditions set in the experiment 4 (comparison with the initial state)

\begin{tabular}{lcc}
\hline & $\begin{array}{c}\text { Inicial } \\
(\boldsymbol{\mu m})\end{array}$ & $\begin{array}{c}\text { Final }(\mathbf{1 2} \mathrm{h}) \\
(\boldsymbol{\mu m})\end{array}$ \\
\hline Media & 5,063 & 30,68 \\
Desviación estándar & 2,463 & 2,031 \\
$\mathrm{~d}_{10}$ & 2,444 & 12,77 \\
$\mathrm{~d}_{90}$ & 11,58 & 69,91 \\
\hline
\end{tabular}



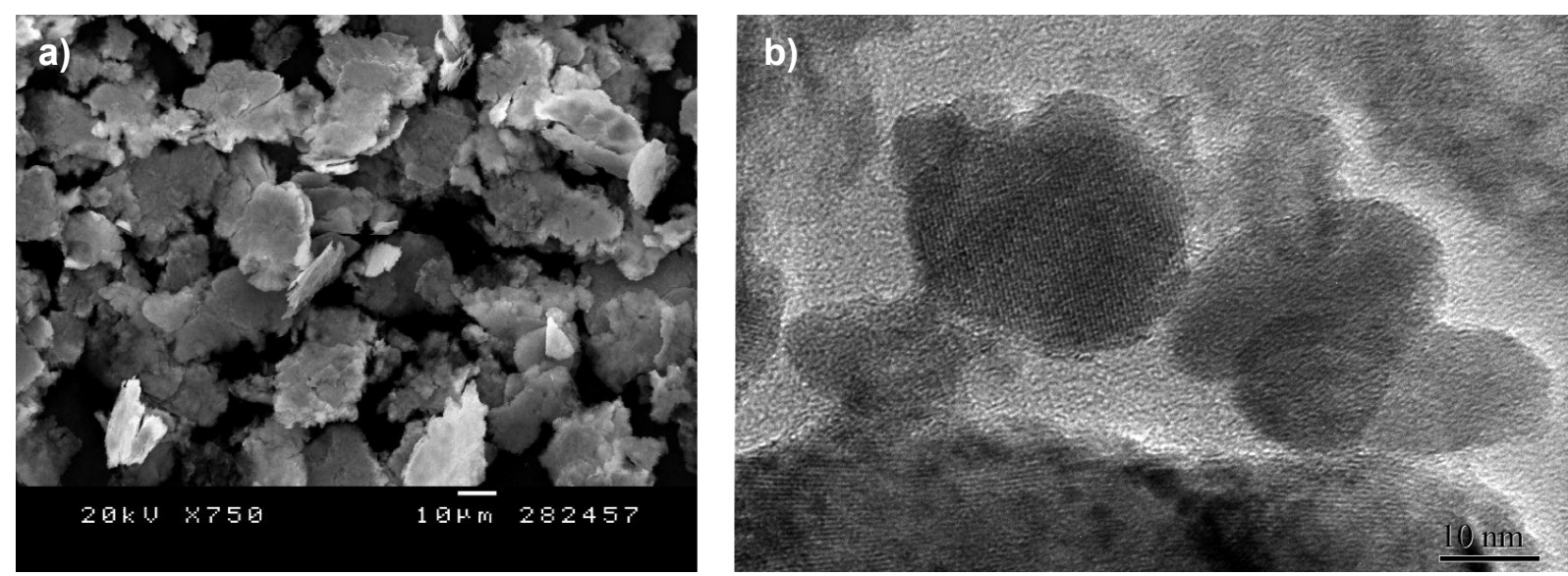

Figura 4. Morfología del polvo molturado en húmedo tras $12 \mathrm{~h}$, según condiciones del experimento 4 , a) SEM y b) TEM.

Figure 4. Powder morphology of the wet mechanically milled powder after $12 h$ according to the $4^{\text {th }}$ experiment set: a) SEM and b) TEM.

de los picos (Fig. 5). En este caso, también se detectó magnetita, con un contenido del $6 \%$. Por tanto, la molienda húmeda consigue reducir la contaminación introducida en la muestra en forma de óxido. Existen estudios, en que precisamente se busca la formación del óxido nanocristalino partiendo de la molturación en agua ${ }^{[12]}$. El tamaño de grano de la muestra del experimento 4 calculado por Williamson-Hall, se estimó del 52,59 nm. Pero la muestra que menor tamaño de grano obtuvo fue la obtenida tras el experimento 1, (29,22 nm): baja velocidad y baja PBR.
Por tanto, según convenga reducir el tamaño de grano o de partícula, habrá que operar de una forma u otra.

\subsection{Molienda criogénica}

El uso de nitrógeno líquido como medio de molturación, ha sido recientemente utilizado por algunos autores con el fin de prevenir la recristalización, favoreciendo la fractura y alcanzando un menor tamaño de grano en tiempos más cortos ${ }^{[13 \text { y } 14]}$.

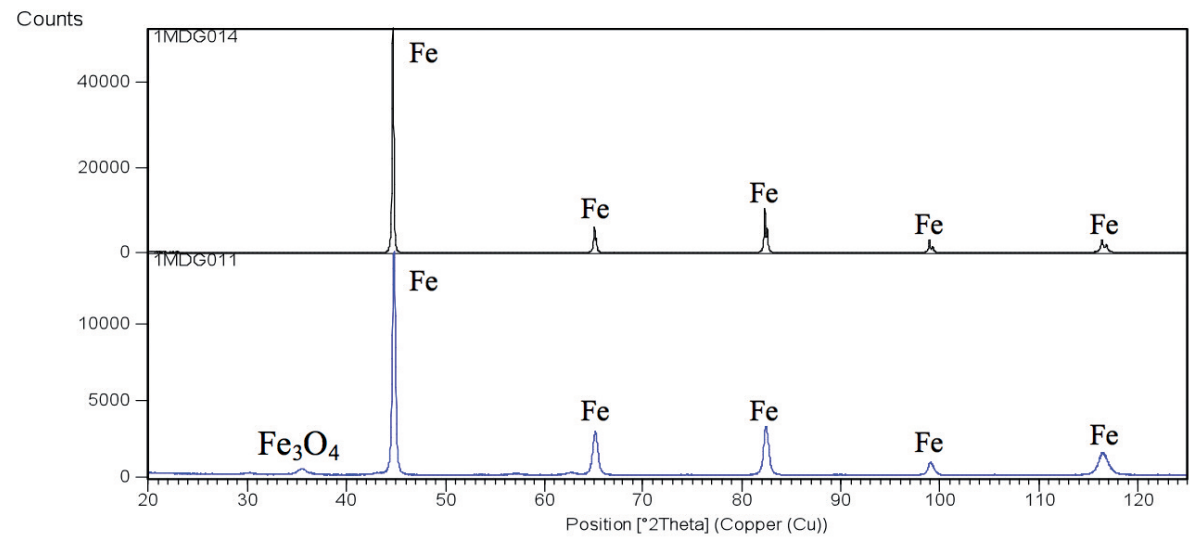

Figura 5. Comparación de los difractogramas obtenidos de la muestra inicial (superior) y la mejor muestra obtenida de la molturación húmeda (inferior).

Figure 5. X-Ray pattern comparison between the unmilled (up) and wet milled (down) Fe powder. 

MECHANICAL MILLING OF A NANOSTRUCTURED DUCTILE IRON POWDER UNDER DRY, WET AND CRYOGENIC ATMOSPHERES

En el presente estudio realizado, tomando muestra hasta 9 h de trabajo, la evolución del tamaño de partícula con el tiempo, es la mostrada en la tabla VIII operando a $600 \mathrm{rpm}, \mathrm{PBR}=1: 20$ y 0,5 \% de ácido esteárico. Como en los casos anteriores, también existe una primera fase de aumento del tamaño de partícula, hasta llegar a alcanzar un máximo y después empieza la reducción.

En la figura 6 a), se observa que existe una gran heterogeneidad; coexisten partículas esféricas con otras de mayor tamaño y de forma un poco más aplanadas.

Ya no se observan partículas excesivamente deformadas, constatando así, la presencia de la fractura, provocada por la fragilización de la muestra al operar en condiciones criogénicas. Observando la figura $6 \mathrm{~b}$ ), se pueden distinguir algunos de los límites de grano, así como la orientación de los planos

Tabla VIII. Datos estadísticos del tamaño de partícula del polvo molturado criogénicamente (comparación con el inicial)

\section{Table VIII. Particle size characteristics of the mechanically milled powder in criogenic conditions (comparison with the initial state)}

\begin{tabular}{lrc}
\hline & $\begin{array}{c}\text { Inicial } \\
(\boldsymbol{\mu m})\end{array}$ & $\begin{array}{c}\text { Final }(9 \mathrm{~h}) \\
(\mu \mathrm{m})\end{array}$ \\
\hline Media & 5,063 & 8,914 \\
Desviación estándar & 2,463 & 3,361 \\
$\mathrm{~d}_{10}$ & 2,444 & 2,350 \\
$\mathrm{~d}_{90}$ & 11,580 & 39,32 \\
\hline
\end{tabular}

cristalinos de muchos de ellos. Los tamaños de grano analizados a partir de las imágenes obtenidas mediante TEM, se encuentran entre 10 y $22 \mathrm{~nm}$, resultando un tamaño promedio de grano de $14,1 \pm 3,3 \mathrm{~nm}$. Por tanto, el tamaño de grano conseguido tras la molturación criogénica y durante la mitad de horas que tras la molturación húmeda, resulta menor.

El ensanchamiento de los picos de difracción de rayos $\mathrm{X}$, indica una reducción del tamaño de grano, a igual que en los medios anteriores (Fig. 7). Además, no se observa la presencia de ningún pico adicional, por lo que la molienda criogénica consigue evitar por completo la oxidación de la muestra.

Mediante el método de Williamson-Hall, se ha calculado que el tamaño de grano estimado para la muestra obtenida tras $6 \mathrm{~h}$ de molturación criogénica, resulta de $33,23 \mathrm{~nm}$.

\section{CONCLUSIONES}

- Una vez optimizadas las condiciones de trabajo, de este estudio se puede concluir que, en cuanto a tamaño de partícula, la molienda seca es más efectiva que la molienda húmeda, habiendo reducido un $43 \%$ el tamaño una vez optimizadas las variables; sin embargo, con la molienda húmeda se consigue una mayor homogeneización de la muestra. La molienda criogénica tras $9 \mathrm{~h}$, obtuvo un mejor resultado que la húmeda.

- Con respecto al tamaño de grano, los tamaños calculados por W-H tras $6 \mathrm{~h}$ de molturación para las 3 técnicas son similares, mientras que tras $12 \mathrm{~h}$ de molturación, la molienda seca ha conseguido
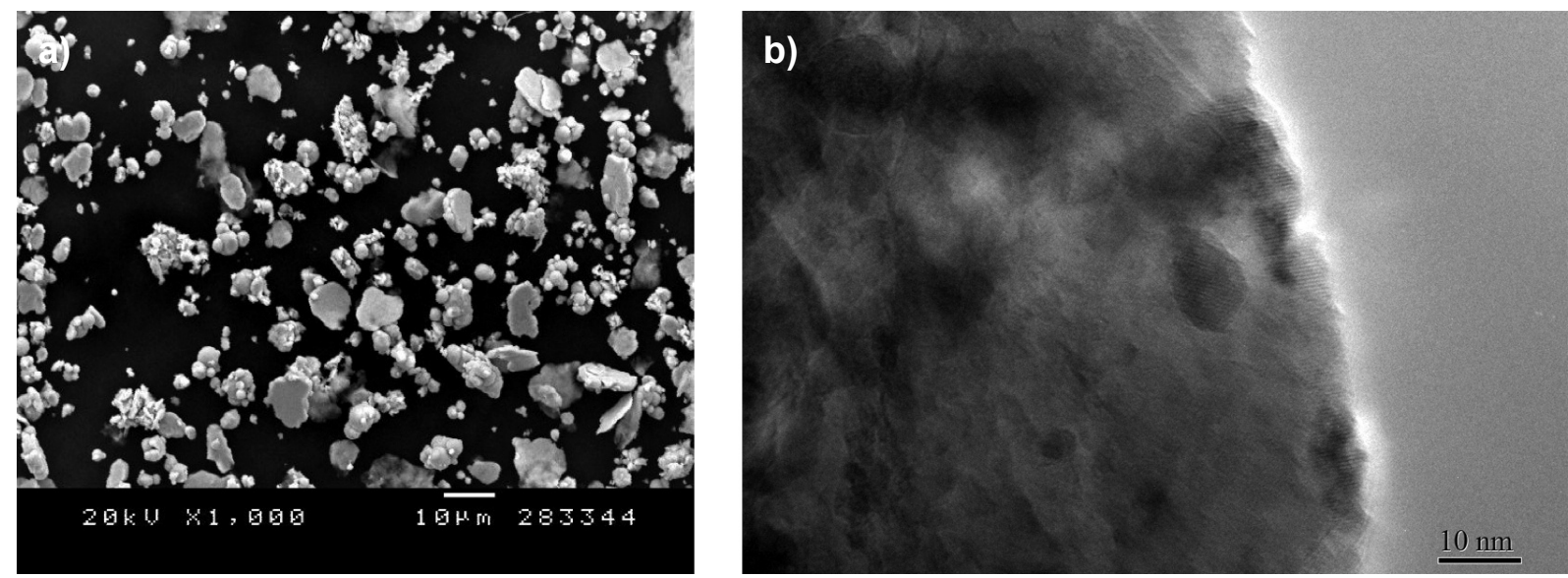

Figura 6. Morfología del polvo molturado criogénicamente a $600 \mathrm{rpm}, \mathrm{PBR}=1: 20$, y $0,5 \%$ de ácido esteárico, a) tras $9 \mathrm{~h}$ (imagen SEM) y b) tras $6 \mathrm{~h}$ (imagen TEM).

Figure 6. Powder morphology of the cryogenic mechanically milled powder at $600 \mathrm{rpm}$, $P B R=1: 20$, and $0.5 \%$ of stearic acid, after: a) $9 \mathrm{~h}$ SEM and b) $6 \mathrm{~h}$ TEM. 


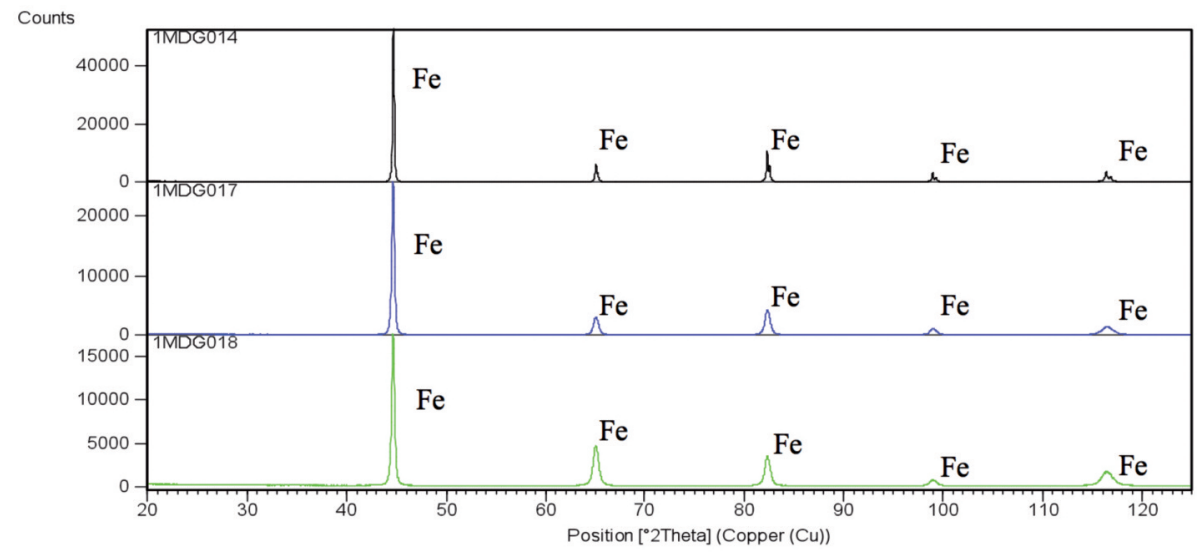

Figura 7. Comparación de los difractogramas obtenidos de la muestra inicial (superior) y las muestras obtenidas de la molturación criogénica (inferior) $3 \mathrm{~h}$ y $6 \mathrm{~h}$.

Figure 7. X-Ray pattern comparison between the unmilled (up) and cryomilled (down) $3 \mathrm{~h}$ and $6 \mathrm{~h}$, Fe powder.

una reducción del $62 \%(24,63 \mathrm{~nm})$ y la húmeda del $55 \%(29,22 \mathrm{~nm})$ y, finalmente, a partir de las imágenes de TEM, tras $12 \mathrm{~h}$ de molienda seca se observan granos entorno a los $11,8 \pm 3,9 \mathrm{~nm} \mathrm{y}$ $16,5 \pm 5,9 \mathrm{~nm}$ en molienda seca y húmeda respectivamente, mientras que tras $6 \mathrm{~h}$ de molturación criogénica, los granos oscilan alrededor de $14,1 \pm 3,3 \mathrm{~nm}$.

- Por último, cabe también destacar, que la molienda en medio criogénico consigue la formación de una atmósfera protectora, evitando por completo la oxidación del polvo de hierro molturado, a diferencia de lo que sucede en la molturación en seco y en húmedo.

\section{Agradecimientos}

Los autores quieren agradecer a la Generalitat de Catalunya por el proyecto 2009SGR390.

\section{REFERENCIAS}

[1] L. Austin y F. Concha: "Diseño y simulación de circuitos de molienda y clasificación", Universidad Técnica Federico Santa María Ediciones, $1^{\mathrm{a}}$ Ed., Chile 1994, pp. 1-174.

[2] A. Zaluska, L. Zaluski y J.O. Ström-Olsen, J. Alloy. Compd. 288 (1999) 217-225.
[3] D.L. Huber, Small 5 (2005) 482-501.

[4] L. Li, M. Fan, R.C. Brown, J. (Hans) Van Leeuwen, J. Wang, W. Wang, Y. Song y P. Zhang, Critical Reviews, Environ. Sci. Technol. 36 (2006) 405-431.

[5] B. Cullity, Introduction to magnetic materials, Addison-Wesley, Reading, Massachussetts: Addison-Wesley Publishing Company (1972).

[6] M.E. McHenry, M.A. Willard y D.E. Laughlin, Prog. Mater. Sci. 44 (1999) 291-433.

[7] K. Takadate, A. Kojima, A. Makino y A. Inoue, Scripta. Mater. 44 (2001) 1.401-1.405.

[8] S. Takaki, K. Kawasaki e Y. Kimura, J. Mater. Proc. Technol. 117 (2001) 359-363.

[9] T.R. Malow y C.C. Koch, Metal. Mater. Trans. A, 29A (1998) 2.285-2.295.

[10] J.J. Suñol, A. González, L. Escoda y M.T. Mora, Rev. Metal. Madrid 44 (2008) 216-221.

[11] C. Suryanarayana, Prog. Mater. Sci. 46 (2001) $1-184$

[12] D. Chen, S. Ni y Z. Chen, China Particuology 5 (2007) 357-358.

[13] E.J. Lavernia, B.Q. Han y J.M. Schoenung, Mater. Sci. Eng. A 493 (2008) 207-214.

[14] D.B. Witkin y E. J. Lavernia, Prog. Mater. Sci. 51 (2006) 1-60.

[15] L. Cabrera, S. Gutierrez, N. Menéndez, M.P. Morales, P. Herrasti, Magnetite nanoparticles: Electrochemical synthesis and characterizacio, Electrochemica, Acta 53, 8 (2008) 3.436-3.441. 